Provided for non-commercial research and education use. Not for reproduction, distribution or commercial use.

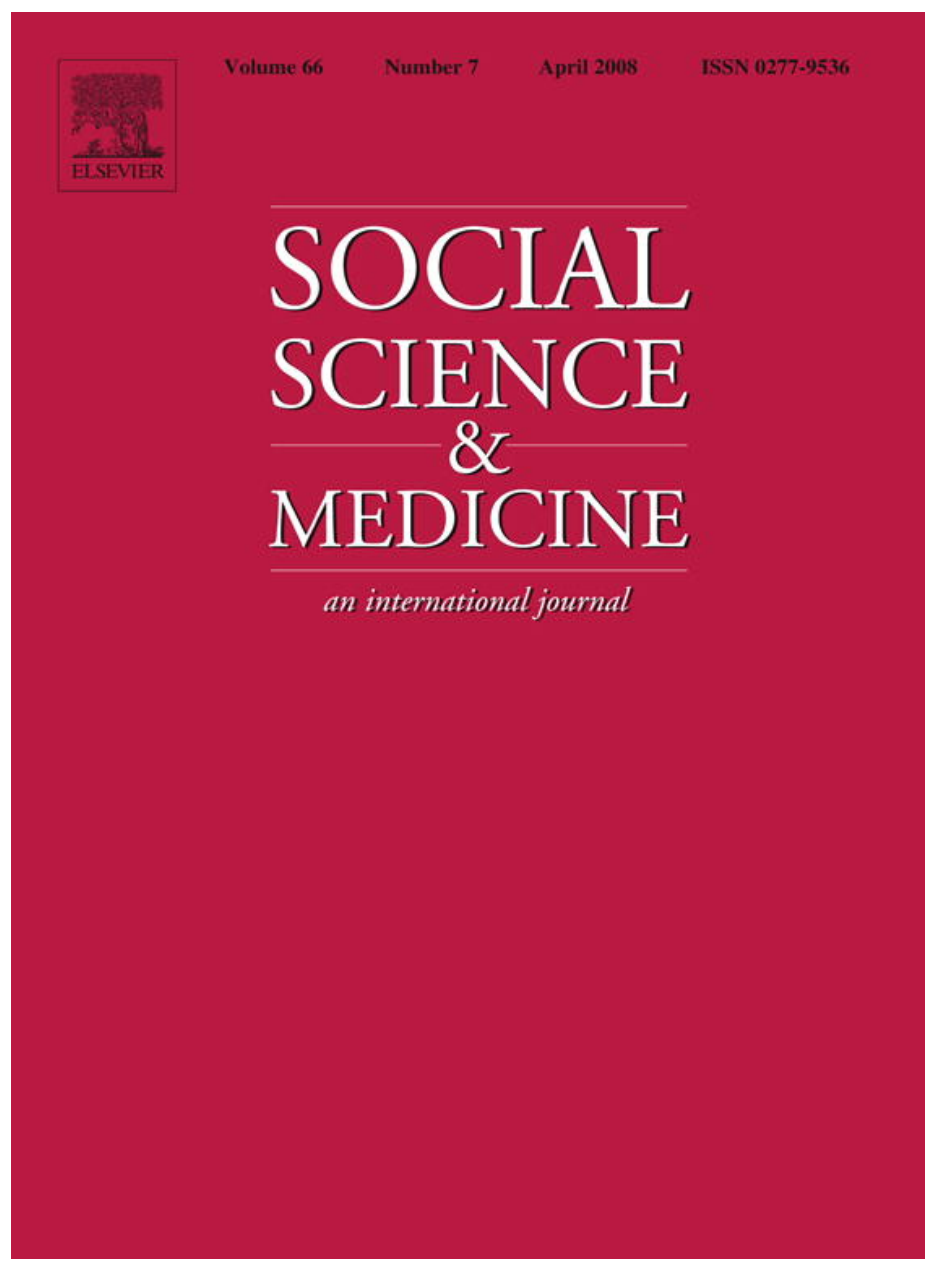

This article was published in an Elsevier journal. The attached copy

is furnished to the author for non-commercial research and education use, including for instruction at the author's institution, sharing with colleagues and providing to institution administration.

Other uses, including reproduction and distribution, or selling or licensing copies, or posting to personal, institutional or third party websites are prohibited.

In most cases authors are permitted to post their version of the article (e.g. in Word or Tex form) to their personal website or institutional repository. Authors requiring further information regarding Elsevier's archiving and manuscript policies are encouraged to visit: 


\title{
Performing stable angina pectoris: An ethnographic study ${ }^{\text {it }}$
}

\author{
Claire Somerville ${ }^{\mathrm{a}}$, Katie Featherstone ${ }^{\mathrm{b}}$, Harry Hemingway ${ }^{\mathrm{c}}$, \\ Adam Timmis ${ }^{\mathrm{d}}$, Gene Solomon Feder ${ }^{\mathrm{e}, *}$ \\ ${ }^{a}$ Queen Mary University of London, London, UK \\ ${ }^{\mathrm{b}}$ Cardiff University, Cardiff, Wales \\ ${ }^{\mathrm{c}}$ University College London, London, UK \\ ${ }^{\mathrm{d}}$ Barts and the London NHS Trust, Whitechapel, London, UK \\ ${ }^{\mathrm{e}}$ Bristol University, Bristol, UK
}

Available online 30 January 2008

\begin{abstract}
Symptoms play a crucial part in the formulation of medical diagnoses, yet the construction and interpretation of symptom narratives is not well understood. The diagnosis of angina is largely based on symptoms, but a substantial minority of patients diagnosed with "non-cardiac" chest pain go on to have a heart attack. In this ethnographic study our aims were to understand: (1) how the patients' accounts are performed or enacted in consultations with doctors; (2) the ways in which ambiguity in the symptom narrative is managed by doctors; and (3) how doctors reach or do not reach a diagnostic decision. We observed 59 consultations of patients in a UK teaching hospital with new onset chest pain who had been referred for a specialist opinion in ambulatory care. We found that patients rarely gave a history that, without further interrogation, satisfied the doctors, who actively restructured the complex narrative until it fitted a diagnostic canon, detaching it from the patient's interpretation and explanation. A minority of doctors asked about chest pain symptoms outside the canon. Re-structuring into the canonical classification was sometimes resisted by patients who contested key concepts, like exertion. Symptom narratives were sometimes unstable, with central features changing on interrogation and re-telling. When translation was required for South Asian patients, doctors considered the history less relevant to the diagnosis. Diagnosis and effective treatment could be enhanced by research on the diagnostic and prognostic value of the terms patients use to describe their symptoms.
\end{abstract}

(C) 2008 Elsevier Ltd. All rights reserved.

Keywords: UK; Angina diagnosis; Ethnography; Chest pain; Narrative; Symptoms; Decision-making; Doctor-patient interaction

\footnotetext{
is This study was funded by the NHS Service Delivery and Organisation R\&D Programme and the Department of Health. Harry Hemingway is supported by a Public Health Career Scientist Award from the Department of Health.

* Corresponding author. Bristol University, Unit of academic primary health care, 25 Belgrave Road, Bristol BS8 2AA. Tel.: +44(0)11773313865.

E-mail addresses: c.somerville@qmul.ac.uk (C. Somerville), featherstonek@cardiff.ac.uk (K. Featherstone), h.hemingway@ucl. ac.uk (H. Hemingway), adamtimmis@mac.com (A. Timmis), gene. feder@bristol.ac.uk (G.S. Feder).
}

\section{Introduction}

Understanding the classification of disease (Sinding, 1989; Wailoo, 1997) and medical diagnoses (Featherstone, Latimer, Atkinson, Pilz, \& Clarke, 2005; Mol \& Elsman, 1996) is central to social scientific theories about the construction of medical knowledge (Armstrong, 1983; Atkinson, 1981). Even in an era when clinicians have a glittering array of diagnostic technologies at their disposal, from blood tests to imaging 
methods, the patient history retains a central place in diagnostic decisions. This is the case even in a specialty that avidly embraces new technologies to aid diagnosis: cardiology (Fox, 2005). This paper builds on studies in the fields of anthropology, sociology and history of medicine tracing the development and construction of a medical specialty (Wailoo, 1997), the emergence of clinical concepts (Martin, 1994) and the mutability of diseases (Sinding, 1989; Young, 1997). Here we explore the ways in which the classification of chest pain and identification of angina is accomplished within the clinical specialty of cardiology. The process of symptom classification and disease definition has consequences for patients (Bowker \& Star, 1999), underpinning diagnosis and treatment within clinical practice.

Chronic angina pectoris is one of the most prevalent forms of heart disease. As well as the impact of its symptoms on quality of life, angina increases the risk of acute coronary syndromes and death in women and men (Hemingway et al., 2006). Angina is more common than a heart attack as the initial presentation of coronary disease, hence early diagnosis is important. In the initial diagnosis, clinicians discriminate between patients with underlying coronary artery disease from those with a non-cardiac cause of chest pain using the patient's description of their chest pain, particularly its quality, duration and position. There is a large quantitative literature on angina diagnosis, highlighting the central importance of the patient's history and the more limited, supportive role of non-invasive testing (Fox, 2005; Hill \& Timmis, 2002). The diagnosis of angina can be problematic and the current codification of cardiac and non-cardiac chest pain may miss patients with significant coronary artery disease. In a recent study, we found that a third of fatal and non-fatal acute coronary events after onset of chronic symptoms were in patients who were thought to have non-cardiac chest pain (Sekhri, Feder, Junghans, Hemingway, \& Timmis, 2006a). This mis-classification means that patients are deprived of appropriate lifestyle advice, as well as drugs and coronary revascularization which may improve symptoms of unrecognized coronary disease and prevent coronary events.

Mis-classification of symptoms as non-cardiac may occur for a number of reasons. Group differences in the way that chest pain of cardiac origin is described might contribute to 'missed' diagnoses. For example, women with coronary artery disease describe angina pain differently from men (Philpott, Boynton, Feder, \& Hemingway, 2001) and there are gender-specific differences in risk factors, symptoms and diagnostic approaches (Shaw et al., 2006). The presentation of angina may also differ between ethnic groups. The Rose angina questionnaire performs inconsistently across ethnic groups when compared to electrocardiogram (ECG) findings (Fischbacher, Bhopal, Unwin, White, \& Alberti, 2001).

Our theoretical orientation is phenomenological, focusing on clinical practice. Our perspective is that diseases are performed or enacted in interactions between doctors and patients (Mol, 2002). This perspective "...does not simply grant objects a contested and accidental history (that they acquired a while ago, with the notion of and the stories about their construction), but gives them a complex present too, a present in which their identities are fragile and may differ between sites. It does so by deploying... ethnographic methods of study... describing the various performances - or enactments - of the objects' identities on stage" (Mol, 2002, p. 43).

In this study, we focused on the first act of the performance of angina: the initial "taking" of a history by the doctor from the patient with new onset stable chest pain. By examining how patients are questioned and symptoms articulated, we made no assumptions about how they fit into disease categories. Instead, we focused on how the symptoms and categories of cardiac and noncardiac pain were performed or enacted in the work of a cardiology clinic.

Previous qualitative research examining angina and heart disease has focused on patient beliefs, understanding and attitudes towards cardiovascular health (Emslie, 2005; French, Maissi, \& Marteau, 2005) but has largely ignored the clinical consultation. Those studies that have focused on the consultation have not examined the construction of diagnoses in relation to patient history (Gordon, Street, Kelly, Souchek, \& Wray, 2005). The current emphasis on the history as narrative and as a means of expressing the patient's perspective focuses on the intrinsic value of this perspective rather than its contribution to diagnosis (Haidet \& Paterniti, 2003). To understand how the initial distinction between angina or non-cardiac chest pain is performed or enacted in a cardiology clinic, in this paper we explore the verbal interaction between doctors and patients.

\section{Methods}

Non-participatory observation of a rapid access chest pain clinic was carried out within a UK teaching hospital. Somerville observed one clinical team consisting of three consultant cardiologists, two registrars, four senior house officers, six other junior doctors on short rotations and six technicians. The composition of the 
doctors in the clinic varied from day-to-day, as they had other duties in the hospital. Clinics were observed daily for 3-6 h over a 14-week period in 2004. In addition, the clinical team was observed in less formal settings during lunch and quiet periods in clinic; this allowed the researcher to engage in informal conversation about the mundane work of the cardiology team.

In total, Somerville observed 59 (22 women, 37 men) consultations. Data collection ended when theoretical saturation was achieved for the major themes. Selection criteria for the patient informants included referral for suspected diagnosis of angina, willingness of the patient to participate and the clinical team's agreement. The study was approved by a multi-centre ethics committee (MREC/03/6/019).

Patient consultations varied in duration from $10 \mathrm{~min}$ to $1 \mathrm{~h}$, the majority lasting less than half an hour. The researcher took handwritten near-verbatim field notes during and immediately after the periods of observation. Additional data were gathered from GP referral forms and from conversations between the researcher and the attending doctor, which were directly recorded in field notes. The primary analysis of the data $(41,386$ words) was undertaken by Somerville, Featherstone and Feder. Analysis was iterative and undertaken throughout the research process allowing for categories of themes to be tested with further data collection (Glaser \& Strauss, 1967; Lambert \& Mckevitt, 2002). The text was organized thematically to highlight the performance of symptom classification and possible diagnosis. These themes were discussed with Hemingway and Timmis in relation to cardiovascular clinical epidemiology and to clinical experience, respectively. Through these discussions, themes were sometimes reconfigured and re-tested against the original data.

\section{Work of the clinic}

Diagnosing first presentation chest pain in ambulatory care was the central activity of the rapid access chest pain clinic (Sekhri, Feder, Junghans, Hemingway, \& Timmis, 2006b). Patients attending the clinics were usually referred directly from their GP and often seen within $24 \mathrm{~h}$. About half the patients had their symptoms for less than four weeks and nearly all for less than six months. The patient had an initial consultation with a junior doctor which is the focus of the analysis presented below. All patients had a resting ECG and most also had an ECG recorded while they were walking on a treadmill. About a third of patients saw a second, more senior, doctor (registrar or consultant), who reviewed the initial diagnosis or tried to resolve uncertainty if no diagnosis had been made. If the patient was deemed to have chest pain of "non-cardiac" origin, they were discharged from the clinic without specialist follow up. If the patient was diagnosed with angina, usually couched in terms of "possible" cardiac chest pain, they were referred for follow up in the cardiology outpatient clinic. Within this paper we are particularly concerned with the process by which this central decision was taken.

\section{Results}

In the performance of "cardiac" and "non-cardiac chest pain", the most striking feature of the consultations was the posing and repetition of a limited set of questions which the doctors used to structure the patient narrative. These questions invariably included enquiry about the quality and duration of the pain, followed by precipitating and relieving factors. These corresponded to the features of chronic chest pain (see Table 1) that Diamond and Forrester (1979) recommended for discrimination between patients with a high and low probability of coronary artery disease. Using pooled data from post-mortem and clinical studies in the United States, they made quantitative assessments of diagnostic probability, based on a small number of symptom features combined with age and sex. These symptoms reliably discriminated between patients with chest pain with and without significant coronary artery disease. The ubiquity of these questions in the clinic consultations we observed implies practical utility in distinguishing "cardiac" from "non-cardiac" chest pain. The continuing reference to the DiamondForrester classification in major medical text books and academic journals (Anthony, 2005; Cakir \& Blue, 2007; Sequist, Marshall, Lampert, Buechler, \& Lee, 2006) more than a quarter of a century after its publication gives it a canonical status.

Table 1

Diamond-Forrester classification of chest pain in relation to angina diagnosis

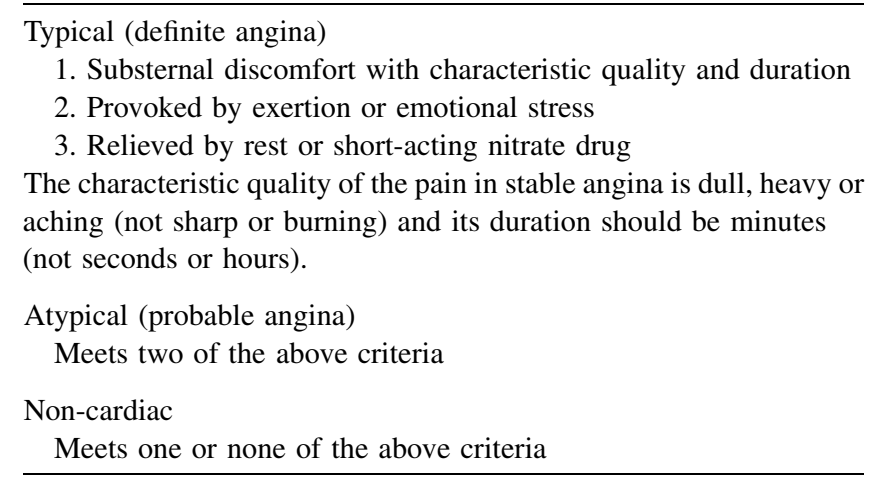




\section{Application of the canon}

We observed four tactics used by doctors to apply this canon in consultations: ignoring contextual detail in the patient narrative, returning repeatedly to the limited set of questions, ignoring some non-canonical presented symptoms and, exceptionally, elicitation of symptoms outside the canon.

\section{Removal of context from narrative}

Consultations mostly started with an open-ended standard statement: "[t]ell me about your pain". The response usually involved a story about the onset of pain containing personal contextual details. The doctors usually did not acknowledge these details, returning repeatedly to the characteristics of the chest pain and quickly made it clear what aspects of the narrative they wanted elaborated. A 63-year-old white man described the occasion when he first experienced symptoms:

Patient: I was on holiday in the Caribbean and drinking heavily. One night I got drunk and went back to my hotel room. I started sweating and got this tingling down my arm. I called reception because I was scared and they got me a doctor. $\mathrm{He}$ said that my blood pressure was up.

Doctor: Have you suffered from this before?

Patient: No, and I don't take any medications. The doctor who saw me said he thought it might be mild angina. He told me to drink lots of water with some sachets in it which he gave me and to get checked out when I got home.

Doctor: Did you have chest pain at the time?

Patient: No. Not really. Years ago I did a treadmill test though.

Doctor: So you had no chest pain at the time?

Patient: No, but it was tight, but I was eating and drinking lots so I don't know if I had pain.

Doctor: When you got the pain were you exerting yourself?

Patient: No, but I was very drunk. You know when you are on holiday you eat lots and get drunk every night.

Doctor: Were you breathless?

Patient: Well, I'm asthmatic but it doesn't effect me much.
[This patient was also seen by the consultant who, after asking the same set of questions again, agreed with the assessment offered by the first doctor, although in addition, the consultant offered some more general advice about excessive drinking]. (RA19. Man, aged 63, white. Diagnosis: non-cardiac)

The context within which the symptoms were first experienced is not considered relevant by the doctor who relentlessly returns to the nature of the pain in pursuit of a diagnosis.

\section{Emphasis on presence or absence of canonical features}

Doctors' questions were largely focused on establishing the role of exertion and a more detailed description of the quality of the pain. In response to the (mostly) closed questions of the doctor, a 71-year-old white woman gave a brief description of her pain that immediately fitted into the diagnostic canon: its precipitation by exertion and the nature of the pain, which was "like someone is getting hold of me" and "squeezing".

Doctor: So you have chest pain?

Patient: Yeah, like someone is getting hold of me. It goes through to the back.

Doctor: And what brings it on?

Patient: Well, soon after I start walking, may be 10 or 15 minutes it goes on for.

Doctor: So it doesn't come on when you are resting?

Patient: No, never, touch wood. It just comes on when I start walking. Sometimes it lasts 30 minutes. It's not strenuous walking- just on the flat. I can feel myself tightening up and all of a sudden I feel it grip. Then, if it stops, it eases off slightly but when I walk again, it starts, like someone squeezing me.

Doctor: Is it sharp or crushing?

Patient: Well that's what I'd call squeezing! It starts at the back.

Doctor: Does it go anywhere?

Patient: No. Now and again I get pain here (points to shoulder), but I think its arthritis.

Doctor: So it stops when you stop walking?

Patient: Yes, when I am indoors I don't get it.

Doctor: How long does it last? 
Patient: All the time I go walking.

[This patient went onto explain that she had been hypertensive for many years and that her daughter, a nurse, came to her home once a week to check her blood pressure. Unusually, the patient asked the doctor about the result of her ECG and appeared to be active in seeking information about her possible diagnosis, asking questions and seeking answers throughout the consultation.] (RA44. Woman aged 71, white. Diagnosis: angina)

This woman went on to have an exercise ECG. Although this was interpreted as normal, the classic description of the pain led the doctor to conclude that she probably had angina and he referred her for coronary angiography.

In addition to the identification of pain characteristics that are positive features within the canon, doctors also actively elicited negative descriptors that indicated a "non-cardiac" diagnosis: chest pain at rest or present all the time, sharp pain or tenderness on the surface of the body, long duration of the pain.

The eliciting of a symptom or sign that is associated with another diagnosis is consistent with applying the diagnostic canon for angina, in so far as their presence is considered by doctors to make angina less likely.

\section{Ignoring symptoms outside the canon}

The corollary of actively eliciting positive and negative features of the canon from patients was the ignoring of other symptoms that are not within the canon, but that may have diagnostic and prognostic significance. The most striking example of this was severity of the chest pain, which is associated with poor prognosis in patients with a diagnosis of angina (Hemingway et al., 2004). Questions about severity only occurred in two of the observed consultations and if patients spontaneously reported severity, this was ignored. For example, in a consultation by a 59 -year-old Bangladeshi woman seen by a senior house officer:

[Daughter translates for her mother. GP referral says that she has 'heavy chest pain radiating to arm, known hypertensive. Her ECG shows inverted Twave'. SHO explains to researcher that this is not significant but does suggest possible ischemia.]

SHO: If she walks does she get pain? Or does her heart shake?

Daughter: It was a couple of months ago.

Patient: 1 year.
SHO: Where is the pain exactly?

Patient: Chest. Back (showing doctor with hand)

SHO: What type of pain is it? Pricking?

Daughter: It's very painful.

SHO: Does she get pain when breathing?

Daughter: Yes, she gets it anytime.

[The three-way nature of this consultation between the doctor, the patient and the daughter meant that the pain narrative was difficult to establish as the patient occasionally interrupted her daughter's translations]. (RA38. Woman, aged 59, Asian. Diagnosis: angina)

Although this patient and her advocate emphasized the severity of her pain, this did not appear to interest the doctor; he did not ask for any elaboration of severity nor functional limitation and swiftly moved on to other symptoms. Four patients spontaneously mentioned severity without any acknowledgement from the doctor. This exemplifies how doctors ignore some aspects of the patient's narrative that are not part of the diagnostic canon.

\section{Eliciting symptoms outside the canon}

We observed that questions about a few noncanonical symptoms, although not asked systematically by all doctors, seemed to contribute to their diagnostic decisions. For example, one doctor repeatedly asked a question about sweating associated with chest pain that was not part of the diagnostic canon. Questions about other symptoms, such as nausea, were sporadically posed by some doctors, but only to some of their patients.

\section{Ambiguity in pain narratives}

We have shown how doctors used a small set of questions to distinguish patients with "cardiac" and noncardiac pain. However, the canon was placed under stress in the face of complex pain narratives. This was reflected in the contestation of "exertion" and other terms, in uncertainty about family history, changes in pain descriptors and the additional complexity of consultations in which English was not the patient's first language. The rehearsing of the pain narrative appeared to be an iterative process, in so far as doctors questioned patients repeatedly about features of the narrative as they came closer to a decision about the identity of the pain. Application of the diagnostic canon was not 
a simple identification of classic descriptors within the patient narrative and then fitting them within the categories of 'cardiac' and 'non-cardiac'. We discuss four areas where the doctors' difficulties are related to the ambiguity of the patient narrative in relation to the canon and how this is addressed by repeated rehearsals and refinement of the patient narrative in the consultation.

\section{Contested terms}

Pain on exertion is a key feature of the canon. Within the observed consultations, patients often could not initially identify precipitating factors for their pain or whether there was any pattern to its recurrence. For example, they often found it difficult to say whether their pain occurred while they were exerting themselves unless they linked it to a specific activity such as climbing stairs or walking to the shops. The interpretation of "exertion" is an example of discordance between doctors and patients around the meaning of key words and phrases related to the canon. For example, when a 52-year-old Bangladeshi man was asked to locate his chest pain:

Patient: It's here. (places his hand onto left side).

Doctor: When did it begin?

Patient: Two weeks ago. Three or 4 months ago I had it here and here (points to one side of his chest then the other with his right hand) and they checked me out and they said I was OK but now I've been sent an appointment for an angiogram at Bart's.

Doctor: Is the pain you have now the same as before?

Patient: No, this time it's here (points to heart) and it moves up here (moves his and up to his left shoulder).

Patient: Do you get it when you walk around?

Doctor: Yes, I get it when I am walking.

[This was followed by a long discussion which contradicts this statement. The patient occasionally raised his eyebrow at questions he felt were irrelevant to his present pain, for example, about where he was from originally. His young son, also present, frequently smiled at the situation as his father created comical responses to the doctor's probes about his pain. After much probing by the doctor, the patient finally denied that he got pain 'on exertion' (the doctor's term). The discussion then moved on to risk factors.] (RA32. 52 year old Bangladeshi man; diagnosis: non-cardiac)

When asked directly whether their pain was exertional, even when patients answered in the affirmative, the additional contextual detail they gave sometimes raised the possibility that the pain could be muscular rather than cardiac in origin. Persistent differences in interpretation of terms and concepts often remained unresolved, although the resulting pain narratives were still used to distinguish cardiac from non-cardiac pain.

\section{Instability of pain narratives}

Compounding the uncertainty about interpretation of terms like "exertion" in the consultation, we found that the pain narrative itself changed on re-telling, particularly when the patient was asked to repeat the narrative by another, usually more senior, doctor, although we discerned no systematic difference in the content and style of questioning as a function of seniority. For example, a 71-year-old white man (RA27), who was first seen by a junior doctor, initially attributed his pain to indigestion and gave a description that suggested noncardiac pain: pain lasting for hours and relieved by indigestion remedies and not occurring while painting his house (suggesting that the pain was not exertional). However, during the course of the consultation his description began to change, partly prompted by comments from his wife and then markedly so when a consultant arrived to review the narrative.

Doctor: Did it go anywhere else?

Patient: No, as soon as I sat down it went.

Doctor: You didn't take painkillers for it?

Patient: No, I took some Gaviscon. Yes, when I got home I took some.

Doctor: And did that help?

Patient: Yes.

[The man was about to be discharged when a consultant puts his head round the curtain and asks if everything is OK. The junior doctor says he is going to get an exercise ECG just to be certain. The consultant asks the patient to recount the story of what has happened. The man repeated the story, he was on his way to Tai Chi and suddenly developed a pain in his chest that lasted a few minutes. The consultant asked if the pain went when he stopped and the man agreed that it did. The story was more concise the second time round and the consultant declared 
that he thought the chest pain was typical - see table 1 - since it came on with exertion and was relieved by rest. The patient did not mention that it was relieved by Gaviscon as he did earlier to the junior doctor and, prompted by his wife who believed her husband was exaggerating the pain, he reduced the time the pain lasted from an hour to just minutes.] (RA27. Man aged 71, white. Diagnosis: angina)

When asked again by the consultant to describe his chest pain, the man's description became more typical of angina: pain occurred while walking and was relieved by rest. These symptoms, plus a positive exercise ECG, led to a diagnosis of angina. Thus, changes in the pain narrative could potentially lead to different diagnoses. From the example above we do not know why the patient's story changed. It is possible that repetition of the story allowed the patient to reflect and clarify details or that the way the consultant posed the questions differed significantly from the junior doctor, to the extent that the patient re-considered and changed his initial responses.

\section{Across languages}

The catchment area of the chest pain clinic included a large south Asian population; almost half of patients seen in the clinic between January 2000 and December 2003 were from one of the south Asian communities. Eleven of the 25 Asian patients observed in this study required interpretation, usually provided by a son or daughter. For example, a 73-year-old Bengali speaker (RA48) was accompanied to the clinic by his son, who translated for his father. When asked about the chest pain, the patient pointed to his chest. His son then explained:

Son: At the moment it is not as bad as it was. He gets pain, and becomes dizzy and sometimes faints. The pain goes up to his head through his neck.

[The doctor asked what the man was doing when he got the pain and the son explained he did not have to be doing anything: '[i]t just comes on when he is sitting'. After some conversation about the nature of the pain the doctor changed tack and tries to establish whether the pain was exertional.]

Doctor: Does it get worse when he walks?

Son: He doesn't walk because he feels dizzy.

Doctor: But does he have pain while he walks?

Son: He does sometimes.

Doctor: How far can he walk?
Son: Not far, he has to stop and rest all the time.

Doctor: Did he get pain when he walked in here this morning?

Son: He had to walk slowly, at a regular pace and then rest.

(RA48. Man aged 73 year, Bengali. Diagnosis: inconclusive at time of observed consultation; referred back for exercise ECG when interpreter was available).

The challenge of incorporating the patient's symptom narrative into the canon is heightened by the limitations on interaction when the doctor needs to communicate via an interpreter and by the loss of precision when hearing a translation of the patient's account. Consultations between doctors and patients who did not speak the same language highlighted the central role of the pain narrative in reaching a diagnosis of angina or non-cardiac pain. These consultations were often longer and seemed less conclusive; more reliance appeared to be placed on the results of the exercise ECG to make a diagnosis. This was also apparent both from informal interviews with the clinical team and from direct observation of consultations. In an interview with a consultant, he acknowledged less reliance on the patient's narrative when they did not speak English because of the difficulty in interpreting their symptoms.

Consultant: Well the language is a problem; there is no doubt about it because when you have a translator it's different. The way that Bengali people express pain I am sure is different to the way others do, so even if you have a translator and you are sure and they say 'arhh, yes, the chest pain is sharp' I am not sure what they mean by sharp is the same as we mean by sharp - and so I think you are much more test-based. Well certainly my decisions are. (DRC02)

Overall, our exploration of ambiguity in patient narratives shows that, despite the apparent simplicity of the pain descriptors in the diagnostic canon, its application to narratives is complex. The contested nature of some of the terms doctors use, their interpretation of missing information, the changes in the narratives on re-telling and the challenge of doctors not speaking a patient's language reflect this complexity.

\section{Diagnostic decision}

We have shown how chest pain narratives are performed or enacted within the consultation and the 
tension between these emergent narratives and the diagnostic canon. Despite this tension, the aim of the clinic is to reach a decision on whether chest pain is likely to be cardiac (angina) or non-cardiac (unrelated to the heart) and this decision was made for all patients. Here we discuss the four main techniques we identified the doctors employed to make a decision on the identity of the chest pain in the face of narratives that were difficult to reconcile with the initial diagnostic canon.

\section{Asymmetry of diagnostic certainty}

We observed that the doctors were more definite about their decision when excluding a cardiac cause. For example, a 41-year-old south Asian man described pain that he had had for several weeks which was made worse when he pressed his hand against his heart.

Doctor: How long does the pain last?

Patient: Maybe 2 hours, but the sharpness comes and goes.

[The doctor paused in his questioning to summarize the description and then continued.]

Doctor: What brings it on? Does it get worse when you are walking or doing chores?

Patient: No, it comes in the evening when I'm not doing anything.

[The doctor quickly concluded that this pain was non-cardiac.] (RA05. Man aged 42, Asian. Diagnosis: non-cardiac)

However, when the doctors diagnosed cardiac chest pain they were less definite, referring the patients for further investigation, in particular, coronary angiography or cardiac perfusion scans. For example, a middle aged black woman (W017) who had diabetes and hypertension (risk factors for heart disease) and gave mixed cardiac and non-cardiac pain descriptors was sent for an exercise ECG. She was unable to complete the test as her blood pressure increased and it was therefore considered inconclusive. Still uncertain, the doctor decided to prescribe anti-anginal drugs (nitrates and beta blockers) and "bring her back" to an outpatient clinic at a later date to review the response to the medication and to consider angiography. We observed that these decisions were often made from pain narratives with a mixture of cardiac and non-cardiac descriptors and that the same descriptors from different patients could result in different diagnoses.

\section{Trumping of canonical features}

The majority of patients' chest pain narratives was difficult to classify even after detailed questioning by the doctor. An example of the complexity of classifying most patients based on their symptom narratives is the consultation of a 63-year-old white woman (RA26) seen by two doctors.

Patient: Like there is a lump there - (pause) and then like a dull ache. I get anxious so I thought it was that.

1st doctor: How long did it last?

Patient: I don't remember really...not long. I took some Rennies but they didn't seem to work. It's sort of like an uncomfortable feeling in my chest. It just doesn't feel right and then I tense up because of my nerves.

1st doctor: Do you get breathless?

Patient: Oh yeah, I can't run for the bus anymore but I smoke so what can I expect?

1st doctor: Does the pain go when you stop?

Patient: Yeah, if I sit down and wait a while it goes.

The patient went on to describe her pain as occurring mainly after food, supporting her own suspicion that the cause was indigestion. The pre-registration doctor is unable to make a diagnosis and asks a senior house officer to review this patient with him.

2nd doctor: So I understand that you only get the pain after eating?

Patient: Well, mainly I think.

2nd doctor: How long after eating?

Patient: Oh a few minutes.

2nd doctor: Before eating, do you get it?

Patient: No.

2nd doctor: At night when you lie flat do you get it?

Patient: No.

2nd doctor: How would you describe the pain?

Patient: Well, I started to sweat and everything around me went all echo-ey. My friend who was with me said I went white.

2nd doctor: So you associated this with eating?

Patient: Yeah, like eating cucumbers or onions. I know that causes it. 
2nd doctor: Do you get pain when you are walking?

Patient: No, I get breathless.

2nd doctor: Like a weight on your chest?

Patient: Yes, that's it. I grasp for breath.

2nd doctor: What happens when you climb stairs?

Patient: Well, I just can't get enough air; I keep trying to get the air in.

2nd doctor: Any pain in your arms?

Patient: No.

2nd doctor: So would you say you have two different types of pain in your chest? One when you eat and another one when you walk?

Patient: Yes, yeah, that's it. It feels like tightness at the moment.

[The doctor explained to the patient that the cause of her symptoms was difficult to determine since there appeared to be two types of pain and that it was often difficult to distinguish between indigestion and heart pain, but suggested that if the pain worsened she should return to the clinic.] (RA26. Woman aged 63, white. Diagnosis: atypical cardiac, probably non-cardiac)

Although the woman presented a number of canonical features, the doctor highlighted other symptoms that he interpreted as non-cardiac and his tentative diagnosis was not based on a simple mapping of canonical features. We did not have follow up data on patients deemed to have non-cardiac chest pain and therefore could not identify which of these patients turned out to have underlying coronary disease (i.e. were misdiagnosed in their initial consultation). This trumping of canonical features by other chest pain characteristics, leading to a diagnosis of non-cardiac chest pain was not consistent: some patients with mixed features were deemed to have cardiac pain. We were unable to discern what tipped the decision one way or another.

\section{Similar descriptors leading to different diagnoses}

Patients used a variety of words to describe their chest pain and there was substantial overlap between terms used by patients ultimately diagnosed as cardiac and non-cardiac. For example, chest pain described as 'like a needle in my heart' was usually considered by the doctors to denote a non-cardiac diagnosis, such as the consultation of a 49-year-old man from Pakistan
(RA18) who described his pain in these terms and was classified by the doctor as 'clearly non-cardiac'.

Patient: I have pain in every muscle. It feels like a needle in my heart and then it went to my neck. (RA18. Man aged 49, Pakistani. Diagnosis: noncardiac)

However, this phrase was also used by patients who were diagnosed as having angina; for example, in a consultation by a 61-year-old Pakistani man (RA10). The doctor asks him to describe his pain in more detail, particularly its onset:

Doctor: How does it feel?

Patient: It's a pain like someone puts a needle in me.

[The doctor continued to question him about the type of pain he was experiencing; but was unable to make a diagnosis. At that point the consultant came in and noted the renal failure and anaemia and judged that he was more likely to have angina than non-cardiac chest pain.] (RA10. Man aged 61, Pakistani. Diagnosis: angina)

This example again highlights the complexity of diagnosis based on the patient narrative and the central role of judgment by the doctors taking into account consciously or unconsciously - other aspects of the narrative and patient characteristics beyond the explicit diagnostic canon. This was most apparent when registrars or consultants were called in by less experienced doctors who were struggling with a diagnosis.

\section{Discussion}

In this paper, we have examined the first act of the performance or enactment of angina when patients with chest pain encounter a cardiologist. Our aim was to understand the diagnostic process of distinguishing cardiac from non-cardiac chest pain in the context of a rapid access chest pain clinic.

This performance was marked by the asking of a small set of questions by the doctor, to the exclusion of other aspects of the pain narrative initially presented by patients. The questions that doctors asked repeatedly in the chest pain clinic have their origin in an epidemiologically based heuristic, developed by Diamond and Forrester, which appears central to the clinical practice of diagnosing chronic chest pain and has taken on a canonical status. Over quarter of a century after Diamond and Forrester developed their classification of chest pain characteristics (Timmis, 1985), we found that it was systematically applied in clinical decisions 
in the diagnosis of angina. Although the application of this canon was problematic in many of the consultations we observed, every patient narrative was ultimately labeled as non-cardiac or (probably) cardiac.

Within these consultations, the doctors initiated a process of re-structuring and reformulating patients' initial accounts of their symptom characteristics, attempting to fit the evolving narrative within a narrow set of canonical categories. This process focused on interpreting the patient's description and explanation of their pain in an attempt to identify and detach from it the core characteristics of the canon. Thus, the personal historical context of the patient's initial narratives was generally ignored, as were symptoms that fell outside of the diagnostic canon. However, it was notable that on some occasions, patients were questioned about non-canonical symptoms, such as pain severity and associated sweating.

Patients sometimes actively resisted the re-structuring of their initial account into the canonical classification by contesting key concepts, such as exertion. Moreover, the pain narrative often changed dramatically, particularly in dialogue with a second, usually more senior, doctor, to the extent that this led to a different diagnosis. In consultations where a translator was required, the patient's description of their symptoms appeared less central to achieving a diagnosis, and out of these consultations, there appeared to be greater use of non-invasive diagnostic technologies: exercise ECG on site and referral to cardiac ultrasound and radioisotope cardiac scans. Thus, diagnosis in patients with new onset chest pain proved complex, with much of this complexity stemming from the apparent restructuring of the patients' pain narratives to fit within a diagnostic canon that relies on the identification of a small subset of the descriptors and associated symptoms that patients use.

We show that the clinical work of diagnosing angina is not a passive process of fitting symptoms into a classification, but an active performance or enactment of angina or non-cardiac pain through a dialogue about the patient's symptoms, a form of practical interpretative work on the part of the doctor (Atkinson, 1997). This diagnostic work has traditionally been identified as part of the tradition of clinical nosography and the classificatory mentality of medical thinking (Friedson, 1970; King, 1982,). Our findings also confirm Armstrong's recognition of discretion and perception in clinical decision-making (Armstrong, 2002), although our analysis reveals a more constrained process defined by more explicit diagnostic criteria, which we have termed a canon.
The performance of angina and the expertise of the doctor within the clinical work of angina diagnosis includes the articulation of uncertainty and ambiguity, as Featherstone found in the diagnosis of dysmorphology in the clinical genetics clinic (Featherstone, Latimer, Atkinson, Pilz, \& Clarke, 2005). However, an important difference is that within the chest pain clinic, deferral did not appear to be an option and was never utilized by these doctors. Although the positive diagnosis of angina was often tentative, the diagnosis of non-cardiac chest pain was always a definitive disposal of the patient from the clinic.

As far as we can ascertain, this study is unique in examining the consultations of patients with stable chest pain of relatively recent onset, before they had repeated exposure to cardiological evaluation and investigation. By embedding an ethnographer in a clinical team, we were able to collect data from a large number of consultations and from repeated informal contact with the team, allowing a detailed exploration of diagnostic decision-making. In addition, our multidisciplinary research team brought multiple perspectives to the analysis, which we fully exploited through our iterative approach to this analysis (Barry, Britten, Barber, Bradley, \& Stevenson, 1999). However, although all dialogue cited in this paper was transcribed directly from handwritten notes taken during the clinic, our ethnographic methodology precluded audio recording. Thus, we did not have verbatim transcripts of the consultations and potentially missed other terms, subtle nuances or explanatory factors that may have modified our conclusions.

The term "canon", which we have used to describe the current criteria for the diagnosis of angina, is not meant ironically. Criteria for diagnosis are essential in the practice of medicine and have become more formalized across many specialties from rheumatology to psychiatry, although the use of epidemiological data for such purposes is relatively unusual in clinical practice. For example, formal diagnostic criteria in psychiatry (Berrios \& Porter, 1995) or rheumatology are clinically, not epidemiologically, based. There is a sharp contrast between the large evidence base for treatment decisions and that for diagnostic decisions (Mallett et al., 2006). However, the original diagnostic canon for angina was drawn from largely white, male hospital populations in the United States more than half a century ago and is probably too narrow in its application to patients with chest pain. In understanding disease entities as performances or enactments (Mol, 2002), in the first act of the angina performance the doctor works hard to apply a relatively limited script. This turns out to be a complex process, because the patient's initial 
narrative may be difficult to reconcile with this script, even after negotiation and discussion. Nevertheless, the script or canon was ultimately imposed within the consultation. This exemplifies the reduction, the eradication of complexity and "messiness" that characterizes the work of medical classification within clinical settings (Bowker \& Star, 1999). In this study, we have explored how this reduction operates in a chest pain clinic, with patients sometimes not only collaborating in reduction, but also resisting it. In current cardiological practice, the full performance or enactment of angina and associated coronary artery disease entails further investigations, including coronary angiography, which was outside the scope of this study.

Our findings suggest explanations for disagreements on angina diagnosis between physicians and data collected via standardized patient questionnaires (Friedman \& Byington, 1985) and the high inter-rater variability in the interpretation of diagnostic tests (Bindels, Hasman, van Wersch, Pop, \& Winkens, 2003). Although other studies have found gender differences in the presentation of angina pain (Philpott et al., 2001; Shaw et al., 2006), in this study the range of symptoms presented by women and the terms they used to describe them did not differ from those of men. We could not distinguish any differences in diagnosis based on ethnicity, that have been reported elsewhere (Hanna \& Bhopal, 2004) in relation to the Rose angina questionnaire, which could arise from language barriers or the use of a translator.

We know that a substantial minority of patients with new onset chest pain are mis-diagnosed. From the study we have reported here, we suggest that it is possible to identify symptoms within the narrative of patients with new onset chest pain that are currently not part of the angina diagnostic canon. These include severity of stable chest pain, functional impairment or sweating, that may help discriminate between angina and noncardiac chest pain. As part of a renewed interest in research on symptoms, there are two possible routes to further exploration of the diagnosis of stable angina in patients with new onset chest pain. The first would entail further detailed qualitative investigation that examines the transformation of the patient narrative into a clinical history using a more detailed discourse analysis (Sarangi \& Roberts, 1999). The second would be to use natural language processing programmes (Pakhomov et al., 2007) that can be used to analyse the terms patients use to describe their symptoms and link this to other diagnostic measures and future outcomes. Such research could inform medical training about history taking and diagnosis and possible standardization via structured interviews for some conditions. We already know that decision support can improve diagnosis (Kawamoto, Houlihan, Balas, \& Lobach, 2005). In the context of angina, it is possible that formalizing the canon within a decision support programme, particularly if it is expanded with new terms, could overcome the inconsistent questioning and the apparent unpredictable decisions that we observed in the clinic. A randomized trial of decision support for virtual patients with new onset stable chest pain found improved effectiveness of referral for further investigation (Junghans et al., 2007).

On the other hand, it is possible that any standardization, even based on better epidemiological research on the diagnostic and prognostic value of a wider range of symptoms may not be sufficient to improve outcomes for patients because of an irreducible complexity of symptoms. It is possible the current diagnostic method used in the chest pain clinic incorporates tacit knowledge and judgment based on ineffable features of the patients (Gillett, 2006), which might be obliterated by decision support systems, resulting in diagnostic and treatment decisions that are less beneficial to the patient. This is an empirical question that can be tested.

\section{References}

Anthony, D. (2005). Diagnosis and screening of coronary artery disease. Primary Care, 32(4), 931-946.

Armstrong, D. (1983). Political anatomy of the body: Medical knowledge in Britain in the twentieth century. Cambridge: Cambridge University Press.

Armstrong, D. (2002). The rise of surveillance medicine. [Reprinted]. In S. Nettleton, \& U. Gustafsson (Eds.), Sociology of health and illness: A reader. Polity Press.

Atkinson, P. (1981). The clinical experience: The construction and reconstruction of medical reality. Farnborough: Gower Press.

Atkinson, P. (1997). The clinical experience: The construction and reconstruction of medical reality, (2nd ed.). Aldershot: Ashgate.

Barry, C., Britten, N., Barber, N., Bradley, C., \& Stevenson, F. (1999). Using reflexivity to optimize teamwork in qualitative research. Qualitative Health Research, 9(1), 26-44.

Berrios, G., \& Porter, R. (Eds.). (1995). A History of Clinical Psychiatry: The Origin and History of Psychiatric Disorders. London: Athlone Press.

Bindels, R., Hasman, A., van Wersch, J., Pop, P., \& Winkens, R. (2003). The reliability of assessing the appropriateness of requested diagnostic tests. Medical Decision Making, 23(1), $31-37$.

Bowker, G., \& Star, S. (1999). Sorting things out: Classification and its consequences. Cambridge, MA: MIT Press.

Cakir, B., \& Blue, K. (2007). How to improve the management of chest pain: hospitalists and use of prediction rules. Southern Medical Journal, 100(3), 242-247.

Diamond, G., \& Forrester, J. (1979). Analysis of probability as an aid in the clinical diagnosis of coronary-artery disease. New England Journal of Medicine, 300(24), 1350-1358. 
Emslie, C. (2005). Women, men and coronary heart disease: a review of the qualitative literature. Journal of Advanced Nursing, 51(4), 382-395.

Featherstone, K., Latimer, J., Atkinson, P., Pilz, D., \& Clarke, A. (2005). Dysmorphology and the spectacle of the clinic. Sociology of Health and Illness, 27, 551-574.

Fischbacher, C., Bhopal, R., Unwin, N., White, M., \& Alberti, K. (2001). The performance of the Rose angina questionnaire in South Asian and European origin populations: a comparative study in Newcastle, UK. International Journal of Epidemiology, 30(5), 1009-1016.

Fox, K. (2005). Investigation and management of chest pain. Heart, 91(1), 105-110.

French, D., Maissi, E., \& Marteau, T. (2005). The purpose of attributing cause: beliefs about the causes of myocardial infarction. Social Science \& Medicine, 60(7), 1411-1421.

Friedman, L., \& Byington, R. (1985). Assessment of angina pectoris after myocardial infarction: comparison of "Rose Questionnaire" with physician judgement in the beta-blocker heart attack trial. American Journal of Epidemiology, 121(4), 555-562.

Friedson, E. (1970). Profession of medicine: A study of the sociology of applied knowledge. New York: Dodd, Mead.

Gillett, G. (2006). Medical science, culture, and truth. Philosophy, Ethics, and Humanities in Medicine, 1(1), 13-23.

Glaser, B., \& Strauss, A. (1967). The discovery of grounded theory. New York: Aldine.

Gordon, H., Street, R., Kelly, P., Souchek, J., \& Wray, N. (2005). Physician-patient communication following invasive procedures: an analysis of post-angiogram consultations. Social Science \& Medicine, 61(5), 1015-1025.

Haidet, O., \& Paterniti, D. (2003). "Building" a history rather than "taking” one. Archives of Internal Medicine, 163, 1134-1140.

Hanna, L., \& Bhopal, R. (2004). Assessing cultural and linguistic appropriateness of the Rose angina questionnaire in three ethnic groups. Ethnicity \& Health, 9, S12-S13.

Hemingway, H., Fitzpatrick, N., Gnani, S., Feder, G., Walker, N., \& Crook, A., et al. (2004). Prospective validity of measuring angina severity with Canadian Cardiovascular Society class: the ACRE study. Canadian Journal of Cardiology, 20(3), 305-309.

Hemingway, H., McCallum, A., Shipley, M., Manderbacka, K., Martikainen, P., \& Keskimaki, I. (2006). Incidence and prognostic implications of stable angina pectoris among women and men. The Journal of the American Medical Association, 295(12), 1404-1411.

Hill, J., \& Timmis, A. (2002). Exercise tolerance testing. British Medical Journal, 324, 1084-1087.

Junghans, C., Feder, G., Timmis, A. D., Eldridge, S., Sekhri, N., \& Black, N., et al. (2007). Effect of patient-specific ratings vs conventional guidelines on investigation decisions in angina: appropriateness of referral and investigation in angina (ARIA) trial. Archives of Internal Medicine, 167(2), 195-202.

Kawamoto, K., Houlihan, C., Balas, A., \& Lobach, D. (2005). Improving clinical practice using clinical decision support systems: a systematic review of trials to identify features critical to success. British Medical Journal, 330, 765-773.

King, L. (1982). Medical thinking: A historical preface. Princeton University Press.
Lambert, H., \& Mckevitt, C. (2002). Anthropology in health research: from qualitative methods to multidisciplinarity. British Medical Journal, 325, 210-213.

Mallett, S., Deeks, J., Halligan, S., Hopewell, S., Cornelius, V., \& Altman, D. (2006). Systematic reviews of diagnostic tests in cancer: review of methods and reporting. British Medical Journal, 333(7565), 413-420.

Martin, E. (1994). Flexible bodies: Tracking immunity in American culture from the days of polio to the age of AIDS. Beacon Press.

Mol, A. (2002). The body multiple: Ontology in medical practice. Durham, NC: Duke University Press.

Mol, A., \& Elsman, B. (1996). Detecting disease and designing treatment. Duplex and the diagnosis of diseased leg vessels. Sociology of Health and Illness, 18(5), 609-631.

Pakhomov, S., Hemingway, H., Weston, S., Jacobsen, S., Rodeheffer, R., \& Roger, V. (2007). Epidemiology of angina pectoris: role of natural language processing of the medical record. American Heart Journal, 153(4), 666-673.

Philpott, S., Boynton, P., Feder, G., \& Hemingway, H. (2001). Gender differences in descriptions of angina symptoms and health problems immediately prior to angiography: the ACRE study. Appropriateness of Coronary Revascularisation study. Social Science \& Medicine, 52(10), 1565-1575.

Sarangi, S., \& Roberts, C. (Eds.). (1999). Talk, work and institutional order: Discourse in medical, mediation and management settings. Berlin: Mouton de Gruyter.

Sekhri, N., Feder, G., Junghans, C., Hemingway, H. J., \& Timmis, A. D. (2006a). How effective are rapid access chest pain clinics? Prognosis of incident angina and non-cardiac chest pain in 8762 consecutive patients. Heart, 93(4), 458-463.

Sekhri, N., Feder, G. S., Junghans, C., Hemingway, H., \& Timmis, A. D. (2006b). Rapid-access chest pain clinics and the traditional cardiology out-patient clinic. QJM, 99(3), 135-141.

Sequist, T., Marshall, R., Lampert, S., Buechler, E., \& Lee, T. (2006). Missed opportunities in the primary care management of early acute ischemic heart disease. Archives of Internal Medicine, 166, 2237-2243.

Shaw, L., Bairey Merz, C., Pepine, C., Reis, S., Bittner, V., \& Kelsey, S. (2006). Insights from the NHLBI-Sponsored Women's Ischemia Syndrome Evaluation (WISE) Study: Part I: gender differences in traditional and novel risk factors, symptom evaluation, and gender-optimized diagnostic strategies. Journal of American College of Cardiology, 47(Suppl. 3), S4-S20.

Sinding, C. (1989). The history of resistant rickets: a model for understanding the growth of biomedical knowledge. Journal of the History of Biology, 22(3), 461-495.

Timmis, A. (1985). Probability analysis in the diagnosis of coronary artery disease. British Medical Journal, 291(6507), 14431444.

Wailoo, K. (1997). Drawing blood: Technology and disease identity in twentieth-century America. Baltimore: John Hopkins University Press.

Young, A. (1997). The harmony of illusions: Inventing post-traumatic stress disorder. Princeton University Press. 